\title{
Numerical and Experimental Analysis of Photonic Crystal Fiber Selectively Infiltrated with Silicon Oil
}

\author{
K. Mileńko*, K.A. Rutkowska And T.R. Woliński \\ Faculty of Physics, Warsaw University of Technology, Koszykowa 75, 00-662 Warszawa, Poland
}

\begin{abstract}
In this paper we demonstrate the numerical and experimental investigation of the photonic crystal fiber selectively infiltrated with polymer material, that is a silicon oil DC-704. The results are compared with those obtained for fully infiltrated fiber. The influence of the infiltration on light propagation properties, as well as on temperature sensitivity of the fiber are also presented. The infiltration causes the formation of photonic bandgap effect, and in the case of full infiltration the bandgaps shift equal to $50 \mathrm{~nm}$ is observed due to the change in external temperature in the range of about $40^{\circ} \mathrm{C}$ (i.e. from 24 to $61^{\circ} \mathrm{C}$ ).
\end{abstract}

DOI: $10.12693 /$ APhysPolA.124.589

PACS: 42.81.-i, 42.70.Qs, 42.81.Pa

\section{Introduction}

Photonic crystal fibers (PCFs) are special optical fibers with periodically arranged air channels in the cladding [1]. In such photonic structure propagation of light depends on the geometry and structural parameters of the fiber - therefore PCFs can guide light by two different mechanisms. The index guiding appears when the solid core has higher refractive index than the cladding and the light is confined by the modified total internal reflection (mTIR). In the bandgap PCFs the light is confined in the core with lower refractive index by reflections from the photonic crystal located in the cladding. The photonic bandgap effect can be obtained in PCFs with hollow core, but also in all solid fibers [2]. The structure of the PCFs allows for the air channels to be infiltrated with liquid materials such as liquid crystals (LCs) [3-5], alcohol [6] and polymers [7]. In this way the new class of fibers can be obtained. It plays an important role in sensing applications since the infiltration may significantly enhance sensitivity of the fiber to external physical parameters. The selective infiltration of the PCFs, in a sense that only selected air channels in the fiber are filled with desired liquid, gives the possibility to generate devices with new properties and possible applications. The latter includes such fields of interest including nonlinear optofluidics [8], refractive index [9], high birefringence [10] and temperature sensing [11].

In this paper we present both theoretical and experimental analysis of the PCF infiltrated with silicon oil DC-704. We compare the influence of both full and selective infiltration on the fiber propagation properties. The selective infiltration can be practically achieved by blocking part of the air holes in the fiber facet, in a chosen pattern, with a UV curable glue. The possibility to control the position of the band gaps with external temperature is also presented.

*corresponding author; e-mail: milenko@if.pw.edu.pl

\section{Materials}

Photonic crystal fiber that was used in experimental tests was a large mode area (LMA) 25 fiber, fabricated by NKT Photonics, Denmark. Figure 1 shows the cross-section of the fiber which is an air-silica isotropic PCF with a solid core and six rings of hexagonally distributed air holes. Fiber cladding diameter is $258 \mu \mathrm{m}$, while hole diameter and core diameter are equal to $7.15 \mu \mathrm{m}$ and $25.2 \mu \mathrm{m}$, respectively.

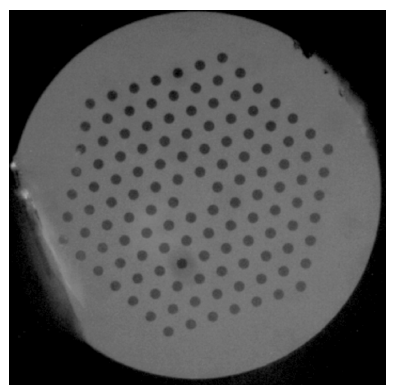

Fig. 1. Picture of the cross-section of employed PCF.

Employed fiber was infiltrated with silicon oil DC-704 (which is $100 \%$ polydimethylsiloxane) with a refractive index of 1.5848 at the wavelength of $550 \mathrm{~nm}$ (at temperature of $20^{\circ} \mathrm{C}$ ). Adventures of using a silicon oil as an infiltration are: low-toxicity, low surface tension, excellent water repellency, and wide range of temperature operation ranging from $-40^{\circ} \mathrm{C}$ to $280^{\circ} \mathrm{C}$.

\section{Infiltration}

Full infiltration of the PCF was obtained by placing one end of photonic crystal fiber in a container with the silicon oil. In this configuration liquid flows into air channels of PCF by capillary forces. The selective infiltration can be achieved by blocking part of the air holes, in chosen pattern, with the UV curable glue (Fig. 2a) [13]. It causes the silicon oil to flow only into unblocked air channels, which form one row in the center of the fiber. After 
the infiltration process, a part of the PCF with the glue was cleaved. The cross-section of the selectively infiltrated fiber is shown in Fig. 2 b, where visible white dots indicate the presence of the silicon oil in the designed air channels.

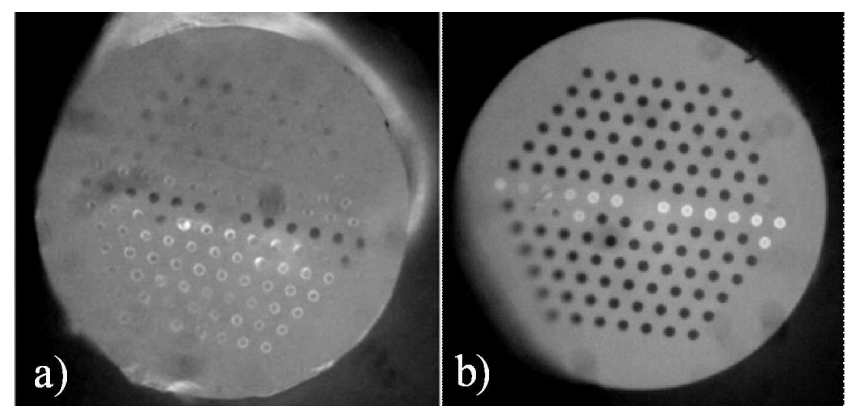

Fig. 2. (a) Photo of PCF selectively covered with glue. (b) PCF after its infiltration with silicon oil and after glue removal.

\section{Numerical analysis}

For the numerical calculations of mode profile we used the RSoft software with a final element method (FEM) applied [14]. Specific values of refractive indices of silica glass and silicon oil used in simulations were approximated and extrapolated by using dispersion relations shown in Fig. 3. Figure 4 shows the numerical results obtained for effective refractive index of the fundamental core mode in the photonic crystal fiber fully infiltrated with silicon oil as a function of the wavelength. There are no core modes in some specific wavelengths which is caused by the formation of the photonic bandgap effect. There are multiple narrow photonic bandgaps and they are densely distributed. This is caused by a high difference in refractive indices of the silica glass and silicon oil.

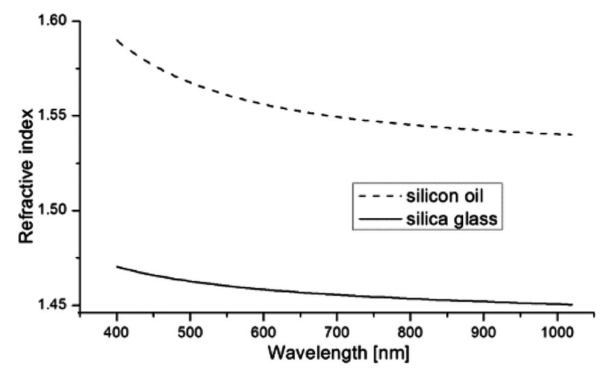

Fig. 3. Dispersion curve for the silicon oil DC-704 [12] and silica glass.

Analogous numerical calculations performed for selectively infiltrated fiber show that for the $x$-polarized fundamental core mode none photonic bandgap effect appears ( $x$ direction is located along the infiltrated air channels). Contrarily, for some specific wavelengths the $y$-polarized fundamental core mode is irradiated or has particularly high propagation losses. For these particular

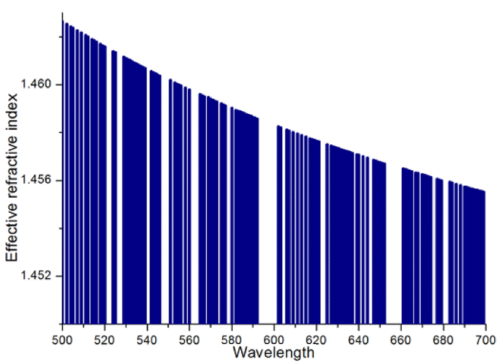

Fig. 4. Effective refractive index for a fundamental core mode of fully infiltrated PCF as a function of wavelength.

wavelengths a core mode is spatially distributed in the infiltrated section of the fiber, i.e. spread along $x$-direction (see Fig. 5b,c). Moreover, as the effective indices of the fundamental core mode are not the same for $x$ - and $y$-polarization (for these specific wavelengths), there is a modal birefringence between both polarizations. Figure 5 shows mode profiles for the $x$ - and $y$-polarized core modes.
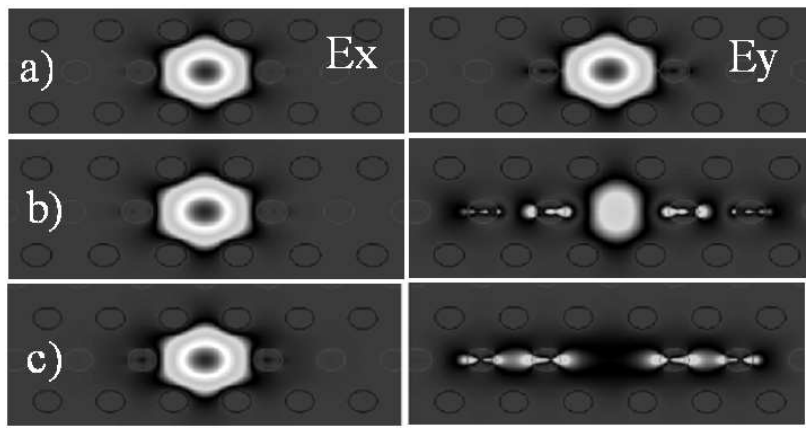

Fig. 5. Mode profiles for $x$ - and $y$-polarizations of the fundamental core modes at three wavelengths: (a) $515 \mathrm{~nm}$, (b) $513 \mathrm{~nm}$, and (c) $552 \mathrm{~nm}$. Please note that only central part of the calculation window is presented.

\section{Experimental results}

Propagation properties of the infiltrated fiber were investigated with the use of the Mikropack Halogen light source with a broadband wavelength range and the Ocean Optics HR4000 spectrometer, while temperature tuning was obtained with the use of the Peltier module. Figure 6 shows the transmission spectrum of the PCF fully infiltrated with the silicon oil. Since the silicon oil has higher refractive index than the silica, the formation of the photonic bandgap effect is observed. The difference in the intensity level between the bandgaps is caused by the light source spectrum. The bandgap formation is consistent with the conducted numerical analysis. The created bandgaps are narrow and densely distributed. The width of the band gap ranges from 25 to $60 \mathrm{~nm}$. 


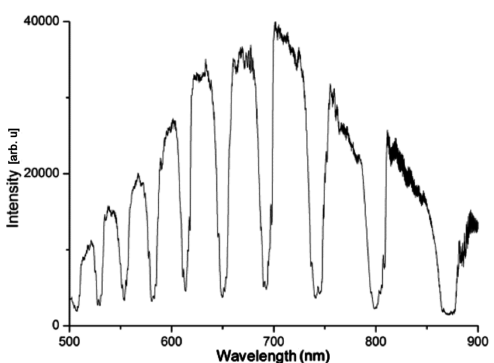

Fig. 6. Measured transmission spectrum of the PCF fully infiltrated with silicon oil DC-704.

Temperature tuning of the bandgaps was investigated by placing the infiltrated fiber on the Peltier module. Temperatures generated in the experiment ranged from $24^{\circ} \mathrm{C}$ to $61^{\circ} \mathrm{C}$. The experimental results show that with an increasing temperature, a blue shift of the photonic bandgaps can be observed (Fig. 7). The total band gap shift equals to $50 \mathrm{~nm}$ in the measured temperature range.

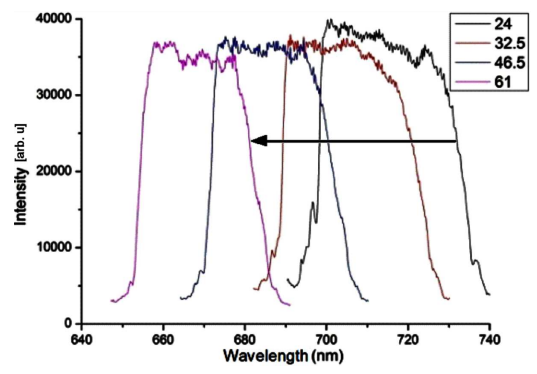

Fig. 7. Temperature tuning of selected band gap for the temperature range of $24-61^{\circ} \mathrm{C}$.

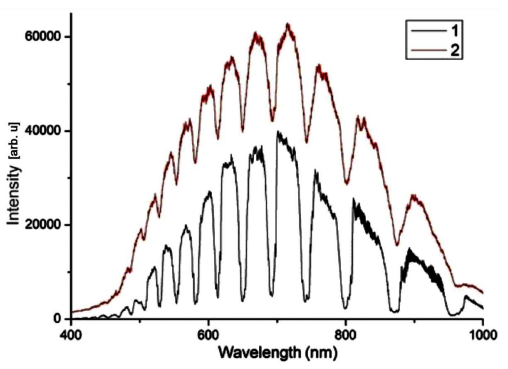

Fig. 8. Measured transmission spectrum of the PCF: 1 - fully and 2 - selectively infiltrated with silicon oil.

Figure 8 shows the measured transmission spectrum of the fully and selectively infiltrated fiber. Specifically, the line no. 2 represents transmission spectrum for the selectively infiltrated $\mathrm{PCF}$, in which the spectral ranges of the higher intensity can be observed. However the light is guided for the remaining wavelengths anyway. This is because the $x$-polarized core modes are guided due to the total internal reflection while for the $y$-polarized ones the core modes are more lost at some specific wavelengths (when not well confined in the fiber core). This is con- sistent with numerical analysis and proves that the fabricated PCF structure, which can guide light with both mTIR and the photonic band-gap effect (depending on degree of infiltaration), shows hybrid propagation.

\section{Conclusion}

In this paper we have presented the photonic crystal fiber infiltrated with the silicon oil DC 704. The formation of photonics bandgaps, as well as their tuning with temperature are presented. Selective infiltration of photonic crystal fibers with polymers gives us possibility to obtain new structures with novel tunable propagation properties. Specifically, by infiltrating one row of the air holes in the center of the photonic crystal fiber, the hybrid operation can be achieved. Selective infiltration of photonic crystal fibers with polymers gives us possibility to obtain new structures with novel tunable propagation properties.

\section{Acknowledgments}

This work was supported by the Polish National Science Centre (NCN) under the grant no. 2011/01/B/ST7/ 05015 .

\section{References}

[1] P.S.J. Russell, Science 299, 358 (2003).

[2] F. Luan, A.K. George, T.D. Hedley, G.J. Pearce, D.M. Bird, J.C. Knight, P.St.J. Russell, Opt. Lett. 29, 2369 (2004).

[3] T. Larsen, A. Bjarklev, D. Hermann, J. Broeng, Opt. Expr. 11, 2589 (2003).

[4] T.R. Woliński, S. Ertman, P. Lesiak, A.W. Domański, A. Czapla, R. Dąbrowski, E. Nowinowski-Kruszelnicki, J. Wójcik, Opto-Electron. Rev. 14, 329 (2006).

[5] M.M. Tefelska, T.R. Woliński, R. Dąbrowski, J. Wójcik, Photon. Lett. Poland 2, 28 (2010).

[6] W. Qian, Ch.L. Zhao, S. He, X. Dong, S. Zhang, Z. Zhang, S. Jin, J. Guo, H. Wei, Opt. Lett. $\mathbf{3 6}$, 3165 (2011).

[7] Ch. Markos, K. Vlachos, G. Kakarantzas, Proc. SPIE 7914, 791427 (2011).

[8] M. Vieweg, T. Gissibl, S.B.T. Pricking, B.T. Kuhlmey, D.C. Wu, B.J. Eggleton, H. Giessen, Opt. Expr. 18, 25232 (2010).

[9] D.K. Wu, B.T. Kuhlmey, B.J. Eggleton, Opt. Lett. 34, 322 (2009).

[10] S. Ertman, T. Nasilowski, T.R. Wolinski, H. Thienpont, Photon. Lett. Poland 1, 13 (2009).

[11] D.J.J. Hu, J.L. Lim, Y. Cui, K. Milenko, Y. Wang, P.P. Shum, T.R. Wolinski, IEEE Photon. J. 4, 1248 (2012).

[12] J.T. Pindera, Techniques of Isodyne Stress Analysis, Springer, Berlin 2001.

[13] K. Milenko, T.R. Wolinski, D.J.J. Hu, J.L. Lim, Y. Wang, P.P. Shum, Photonics Global Conf., PGC 2012, 6458040 (2012).

[14] http://www.rsoftdesign.com/. 